\title{
Multi-Parameter Based Calibration of Long-Link Flexible Manipulators
}

\author{
Zhao Liu ${ }^{1,2}$, Libin Song ${ }^{1,2}$, Xing $\mathrm{Li}^{1 *}$, Bozhao Pan ${ }^{1}$ and Junzhang $\mathrm{Yu}^{1}$ \\ ${ }^{1}$ Tianjin Research Institute for Advanced Equipment, Tsinghua University \\ ${ }^{2}$ Tsingyan Huayu Robot and Automation (Tianjin) Co., Ltd. Tianjin, China \\ ${ }^{*}$ Corresponding author
}

\begin{abstract}
Geometric parameters, reducer stiffness, and link flexibility have significant impacts on the absolute positioning accuracy of the terminals of long-link flexible manipulators. This paper introduces a multi-parameter based calibration method which can improve the absolute positioning accuracy. The experiment was carried out on a 7-DOF long-link flexible manipulator. The pre-analysis involved the stiffness of reducers, the deformation of flexible links, and other structural features of the manipulator. The calibration contained two important steps. The first step provided the approximations of the kinematic parameters and the reduction ratios. The second step focused on the parameter identification of a kinematic error model of the manipulator. This step could improve the accuracy of the kinematic parameters. A laser tracker was used to measure the positions of marked points on the manipulator before and after calibration. The comparison of the measurements obviously shows that the method can improve the absolute positioning accuracy of the manipulator.
\end{abstract}

Keywords-flexible manipulator; calibration; structural deformation; absolute positioning accuracy

\section{INTRODUCTION}

Flexible manipulators are being used in many fields because of various advantages, such as lightweight, flexible to avoid obstacles, be able to work on complex surfaces and high repetitive positioning accuracy. However, the absolute positioning accuracy is low [1], which cannot meet the requirements of absolute accuracy for off-line programming. Therefore, it is important to improve the absolute positioning accuracy of flexible manipulators by calibration.

Calibration is an important technique as a means of improving the absolute positioning accuracy of flexible manipulators. The procedure comprises modeling, measurement, parameter identification and error compensation [2]. The result of calibration essentially depends on the model used for calibration. In terms of the type of model parameters, the calibration can be classified into joint calibration, kinematic calibration and non-kinematic calibration [3]. Kinematic calibration consists of the geometric relation of each joint and the mapping relation from joint space to workspace. Nonkinematic calibration is based on kinematic calibration and integrated with the effect of non-geometric factors, such as mechanical deformation, temperature, backlash etc. [4]

The study of kinematic calibration has been well developed. The kinematic calibration method in domestic is generally based on D-H kinematic model to obtain error mapping model as a means of the identification of geometric parameters and error compensation [5]. Consequently, the absolute positioning accuracy of manipulators can be improved by kinematic calibration. On the other hand, there are a few studies about non-kinematic calibration [6-11]. This is because nongeometric factors are complicated to analyze. Alternatively, it's difficult to establish an independent parameter model since the non-geometric factors are not only coupled with mechanical structure but also the operating environment of manipulators. Nevertheless, non-geometric factors always have a dominant effect on the absolute positioning accuracy in many practical applications, such as long-link spraying robots and large load handling robots. Thus, the calibration and compensation of non-geometric factors are imperative.

The research of this paper was based on a long-link 7-DOF spraying robot. The research focused on the calibration and compensation of such robots which have long links and flexibility. This paper presents a calibration method specifically for such robots. The results of calibration and compensation were analyzed and verified according to the measurement data obtained by a laser tracker.

\section{THE MODELLING OF ROBOT}

The 7-DOF robot has seven revolute joints. The modeling of the kinematics of the robot follows D-H principle. A 3D robot model is shown Figure I.

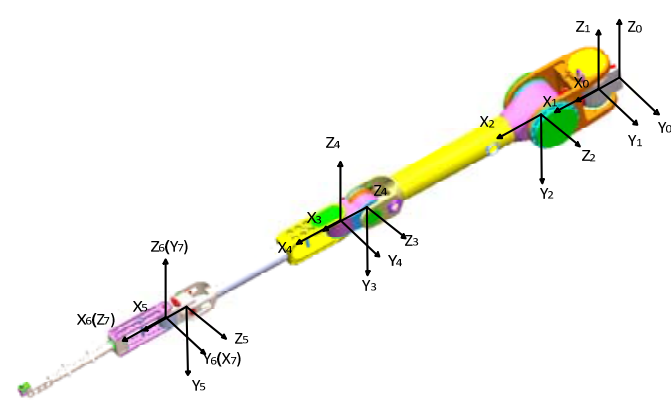

FIGURE I. THE D-H MODEL OF A 7-DOF SPRAYING ROBOT

The first step of $\mathrm{D}-\mathrm{H}$ method is to determine $\mathrm{D}-\mathrm{H}$ parameters: joint angle $\theta_{i}$, link twist $\alpha_{i}$, link offset $d_{i}$, and the link length $a_{i}$. Second, determine the homogeneous transformation matrices between two adjacent links and the homogeneous transformation matrices of each link frame with respect to base coordinate. Finally, determine the homogeneous transformation matrices of the terminal of the robot with 
respect to the base coordinate. The homogeneous transformation matrix $A_{i}$ from link $i$ to link $i-1$ is

$$
\left[\begin{array}{cccc}
\cos \theta_{i} & -\sin \theta_{i} \cos \alpha_{i} & \sin \theta_{i} \sin \alpha_{i} & a_{i} \cos \theta_{i} \\
\sin \theta_{i} & \cos \theta_{i} \cos \alpha_{i} & -\cos \theta_{i} \sin \alpha_{i} & a_{i} \sin \theta_{i} \\
0 & \sin a_{i} & \cos \alpha_{i} & d_{i} \\
0 & 0 & 0 & 1
\end{array}\right]
$$

The homogeneous transformation matrix of the terminal of the 7-DOF robot is calculated by

$$
\mathrm{T}=\mathrm{A}_{1} \mathrm{~A}_{2} \mathrm{~A}_{3} \mathrm{~A}_{4} \mathrm{~A}_{5} \mathrm{~A}_{6} \mathrm{~A}_{7}
$$

\section{THE DEFORMATION ANALYSIS OF REDUCER AND LINK}

\section{A. The Deformation Analysis of Link}

The load can easily cause the deformation of a long flexible link. The deformation includes three aspects: the deformation of the end-face of reducer caused by the overturning force generated by the Centre of Mass (COM) of the link behind the end-face; the deformation of reducer caused by the torsional force generated by the COM of the link behind the reducer; the deformation of link caused by the COM of the link.

The ideas of calibration are as follows:

1) Obtain the distribution of COM of each link and joint at different positions, and the centroid force which leads to the deformation of reducer.

2) Divide the centroid force of each joint into three components along $\mathrm{X}, \mathrm{Y}, \mathrm{Z}$ axes respectively; obtain the overturning moment along $\mathrm{X}$ and $\mathrm{Y}$ axes, the torsional moment along $\mathrm{Z}$ axis, and the moment leads to the deformation of link.

3) Determine the deflection of the end-face of reducer and the deflection of the link based on the moments obtained in 2); substitute the deflection into forward kinematic homogeneous transformation matrix to complete calibration compensation.

\section{B. The Distribution of Centroid Force of Each Joint}

The 3-D model of the robot in Solidworks 2016 can provide the coordinate of a link in joint frame. The coordinate transformation matrix of the COM of the link with respect to the last joint frame can be described by

$$
c_{i}=\left[\begin{array}{cccc}
1 & 0 & 0 & X_{i} \\
0 & 1 & 0 & Y_{i} \\
0 & 0 & 1 & Z_{i} \\
0 & 0 & 0 & 1
\end{array}\right]
$$

The coordinate transformation of the COM of each link, $O_{i}$, in different positions with respect to the base coordinate is calculated by

$$
O_{i}=T_{i-1} * \operatorname{Rot}\left(z, \theta_{i}\right) * c_{i}
$$

The coordinate of the COM of each link is described by

$$
b_{i}=\left[O_{i}(1,4), O_{i}(2,4), O_{i}(3,4)\right]
$$

For example of joint 1 , the gravity of link 1 is calculated by

$$
M_{1}=m_{1}+m_{2}+m_{3}+m_{4}+m_{5}+m_{6}+m_{7}
$$

The coordinate of the resultant gravity of link 1

$$
B_{1}=\left(\sum_{i=1}^{7} b_{i} * m_{i}\right) / M_{1}
$$

Define the vector of the resultant force of link 1 is

$$
V_{1}=\left[\begin{array}{llll}
0 & 0 & -M_{1} & 0
\end{array}\right]^{T}
$$

The position and the vector of the resultant force of link 1 in local coordinate frame is calculated by

$$
\begin{aligned}
& R P_{1}=\left(T_{0} * \operatorname{Rot}\left(z, \theta_{i}\right)\right)^{-1} * B_{1} \\
& R V_{1}=\left(T_{0} * \operatorname{Rot}\left(z, \theta_{i}\right)\right)^{-1} * V_{1}
\end{aligned}
$$

The position and the vector of the resultant force of other links can be also obtained in the same way.

\section{The Deformation Analysis of Reducer}

The deformation due to overturning force is equivalent to the deformation produced by the moment along axes $\mathrm{X}$ and $\mathrm{Y}$, and the deformation produced by the torsional force along axis Z. As shown in Figure II.

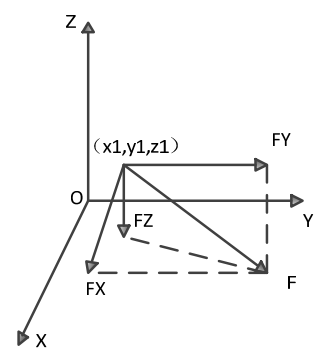

FIGURE II. DECOMPENSATION OF FORCE IN JOINT SPACE

The torque along 3 axes generated by the resultant force of joint are calculated by

$$
\begin{aligned}
& T_{x}=F_{z} * x_{1}-F_{y} * z_{1} \\
& T_{y}=F_{x} * z_{1}-F_{z} * x_{1} \\
& T_{z}=F_{y} * x_{1}-F_{x} * y_{1}
\end{aligned}
$$

The end-faces of reducer and bearing both sustain the overturning moment of the joint as shown in Figure III. 


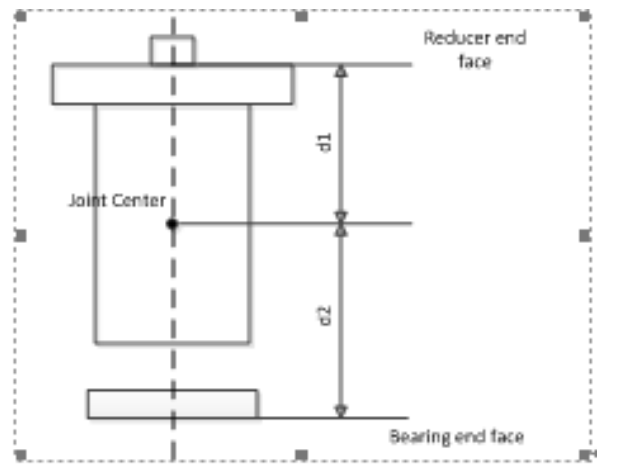

FIGURE III. THE INNER STRUCTURE DIAGRAM OF A JOINT

The overturning moments apply onto the terminal-face of reducer along $\mathrm{X}$, $\mathrm{Y}$ axes can be calculated by

$$
\begin{gathered}
T X=\frac{d_{1}}{d_{1}+d_{2}} T_{x} \\
T Y=\frac{d_{1}}{d_{1}+d_{2}} T_{y}
\end{gathered}
$$

The torsional moment along $\mathrm{Z}$ axis is fixed

$$
T Z=T_{Z}
$$

The overturning stiffness and torsional stiffness are also required for the calculation of overturning angle and torsional angle, conjunct with the overturning moment and the torsional moment. There are seven different reducers equipped on the 7DOF robot. Thus, the overturning stiffness and torsional stiffness of each joint are different. Assume $k_{i a}, k_{i b}$ are the overturning stiffness and torsional stiffness respectively. Thus, the overturning angle and torsional angle can be determined by

$$
\begin{aligned}
& \theta_{i x}=\frac{T X i}{k_{i a}} \\
& \theta_{i y}=\frac{T Y i}{k_{i a}} \\
& \theta_{i z}=\frac{T Z i}{k_{i b}}
\end{aligned}
$$

\section{The Deformation of Link}

It's difficult to measure the stiffness of the links of the robot accurately from independent experiments, because of the inherent tolerance of equipment, the tolerance of machining process and the deformation of reducers. Finite Element Analysis (FEA) method was applied to determine the stiffness of each link. Specifically, the offsets at different home positions of each link were obtained in ANSYS when the gravity of the link was considered simply. This is shown in Figure IV.

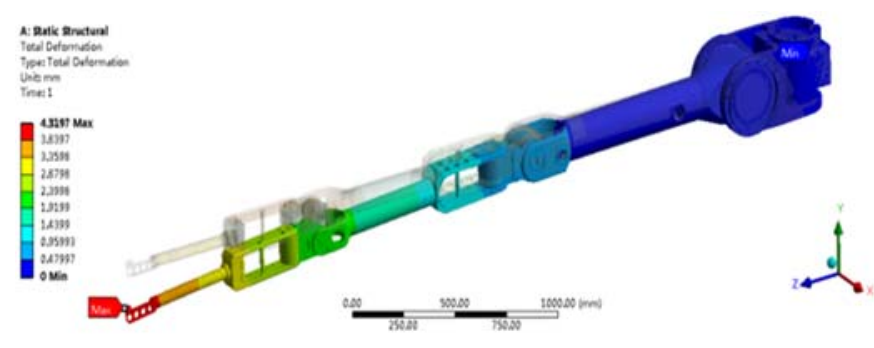

FIGURE IV. STIFFNESS ANALYSIS IN ANSYS

The offsets of the two terminals of a link can be read from the figure above. The difference between two terminals is the deflection of the link, namely $\omega_{i}$. According to the cantilevered beam deflection equation, the stiffness $(k=E I)$ of each link can be calculated by

$$
k_{i}=\frac{T * l_{i}^{2}}{3 * \omega_{i}}
$$

The stiffness of each link is shown in Table I,

TABLE I. THE STIFFNESS OF EACH JOINT

\begin{tabular}{|c|l|l|l|l|l|l|l|}
\hline Link & 1 & 2 & 3 & 4 & 5 & 6 & 7 \\
\hline $\begin{array}{c}\text { Stiffness } \\
/ \mathbf{1 0}^{\mathbf{6}} \mathbf{N m} / \text { rad }\end{array}$ & 148.2 & 621.2 & 132.7 & 211.3 & 466.1 & 0.09 & 0.71 \\
\hline
\end{tabular}

According to the torque obtained in section III, the deflection of each link, which is assumed as cantilevered beam, along each axis is calculated by

$$
\begin{gathered}
\omega_{i X}=\frac{T_{i x * l_{i}}^{2}}{3 * k_{i}} \\
\omega_{i Y}=\frac{T_{i y * l_{i}}^{2}}{3 * k_{i}} \\
\omega_{i Z}=\frac{T_{i z} * l_{i}^{2}}{3 * k_{i}}
\end{gathered}
$$

\section{E. The Compensation of Kinematics}

Calibration compensation aims to compensate the errors of position and pose of robot terminal. The procedure of compensation is: the angle errors and the link deflections in joint space, calculated in the sections above, will be mapped to the terminal of the robot through forward kinematics to obtain practical position and pose of the terminal; compare theoretical value with the practical value to obtain the difference; adding the difference to the theoretical value produces a new set of position and pose; remap the new set of position and pose to joint space to compensate angle errors and link deflections. This is a recursive procedure and it will be done until the error is decreased sufficiently.

The coordinate homogenous transformation, when the deformations for both joint and link are accounted, is 


$$
\begin{gathered}
T_{i}=T_{i-1} * A_{i+1} * \operatorname{Trans}\left(\omega_{i+1}\right) * \\
\operatorname{Rot}\left(x, \theta_{x+1}\right) \operatorname{Rot}\left(y, \theta_{y+1}\right) * \operatorname{Rot}\left(z, \theta_{z+1}\right)
\end{gathered}
$$

Assume the theoretical position and pose of the terminal is described by $T$, the difference between theory and practice is

$$
\Delta T=T-T_{7}
$$

The new pose and position after compensation is therefore

$$
T_{\text {end }}=T+\Delta T=2 T-T_{7}
$$

Substitute the compensated result into the inverse program of the 7-DOF robot will produce the joint angle of each joint.

\section{KINEMATICS CALIBRATION AND COMPENSATION}

\section{A. The Approximate Calibration of Kinematic Parameters and Reduction Ratio}

According to the D-H model of the robot, the length of a link between the central axes of two neighboring joints is described by $a_{i}$, the twist angle between the central axes of two neighboring joints is described by $\alpha_{i} . a_{i}$ and $\alpha_{i}$ are measured by CPA method.

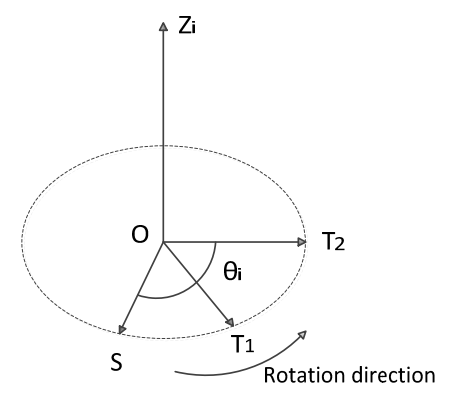

FIGURE V. THE ROTATION OF A JOINT

As shown in Figure $\mathrm{V}$, joint $i$ rotates around its central axis Z. Assume a point, marked at the terminal of the robot, executes a circular trajectory which starts from point $S$; choose another two points $T_{1}$ and $T_{2}$ optionally from the trajectory. Fitting these three points into a curve provides the center of the curve, namely $O$, and the central axis, namely $Z_{i}$. The products of the function between $Z_{i}$ and $Z_{i-1}$ will be $a_{i}$ and $\alpha_{i}$. On the other hand, the deformation due to the overturning of reducer should be compensated to the measurement as a means of eliminating the effect of overturning.

As shown in Figure V, the angle between $\overrightarrow{O S}$ and $\overrightarrow{O T}$ is the joint angle rotate around $Z_{i}$. Thus,

$$
\theta_{d}=\operatorname{acos}\left(\frac{\overrightarrow{U S} * \overrightarrow{V T}}{|\overrightarrow{U S}| *|\overrightarrow{V T}|}\right)
$$

The rotating angle of motor can be calculated by the output of encoder accurately using the equation below

$$
\theta_{e}=2 \pi\left(\frac{\Delta E}{E_{n} * E_{d}}\right)=\frac{\text { abs.displacement }}{32}
$$

Where $\Delta E=E_{T_{2}}-E_{S}$ describes the difference of the outputs of the encoder with respect to $T_{2}$ and $S . E_{n}$ and $E_{d}$ are the resolution and the number of measuring units per revolution of the encoder.

Several positions of the marked point and the corresponding encoder values are recorded while the joints are rotating. The record will be used to calculate $\theta_{e}$ and $\theta_{d}$. A linear function of $\theta_{e}$ and $\theta_{d}$ is estimated by Least Square Method (LSM). The slope of the function is practically the reduction ratio of the reducer.

\section{B. Precise Calibration of Kinematics Parameters}

It's difficult to determine the link offset, $d_{i}$, accurately since the position of the common normal between two adjacent parallel links cannot be measured precisely. Alternatively, it's also difficult to determine the joint angle, $\theta_{i}$, due to inevitable assembling errors. Therefore, an error model of the position and pose of the terminal with respect to $d_{i}$ and $\theta_{i}$ is established. The two parameters can be identified accurately from the model. According to section II, the position and pose of the terminal of the robot is

$$
P=F(a, d, \alpha, \theta)
$$

The approximate calibration discussed in last section provides link length and twist angle. Assume the deviations of link offset and joint angle are $\Delta d, \Delta \theta$ respectively, thus the practical position and pose of the terminal of the robot, $P^{\prime}$, is

$$
\begin{gathered}
P^{\prime}=F(a, d, \alpha, \theta) \\
\Delta P=P-P^{\prime}
\end{gathered}
$$

Equation (31) can be approximated to a linear function when the two deviations are sufficiently small. Namely

$$
\Delta P=\frac{\partial F}{\partial d} \Delta d+\frac{\partial F}{\partial \theta} \Delta \theta
$$

There are seven link offsets and seven joint angles. $\partial d$ represents $\partial d_{1}, \partial d_{2}, \partial d_{3}, \partial d_{4} 、 \partial d_{5}, \partial d_{6}, \partial d_{7}$; and $\partial \theta$ represents $\partial \theta_{1}, \partial \theta_{2}, \partial \theta_{3}, \partial \theta_{4}, \partial \theta_{5}, \partial \theta_{6}, \partial \theta_{7}$. Thus, at an arbitrary position $P_{i}$, the deviations along axes $\mathrm{X}$, $\mathrm{Y}$ and $\mathrm{Z}$ are

$$
\left\{\begin{array}{c}
\Delta P_{i x}=\frac{\partial F_{i x}}{\partial d_{1}} \Delta d_{1}+\cdots+\frac{\partial F_{i x}}{\partial d_{7}} \Delta d_{7}+\frac{\partial F_{i x}}{\partial \theta_{1}} \Delta \theta_{1}+\cdots+\frac{\partial F_{i x}}{\partial \theta_{7}} \Delta d \theta_{7} \\
\Delta P_{i y}=\frac{\partial F_{i y}}{\partial d_{1}} \Delta d_{1}+\cdots+\frac{\partial F_{i y}}{\partial d_{7}} \Delta d_{7}+\frac{\partial F_{i y}}{\partial \theta_{1}} \Delta \theta_{1}+\cdots+\frac{\partial F_{i y}}{\partial \theta_{7}} \Delta \theta_{7} \\
\Delta P_{i z}=\frac{\partial F_{i z}}{\partial d_{1}} \Delta d_{1}+\cdots+\frac{\partial F_{i z}}{\partial d_{7}} \Delta d_{7}+\frac{\partial F_{i z}}{\partial \theta_{1}} \Delta \theta_{1}+\cdots+\frac{\partial F_{i z}}{\partial \theta_{7}} \Delta \theta_{7}
\end{array}\right.
$$

Assume the number of the points measured is $n$, therefore

$$
A \Delta X=b
$$

Where 


$$
\begin{aligned}
& A=\left(\begin{array}{cccc}
\frac{\partial F_{1 x}}{\partial d_{1}} \ldots \frac{\partial F_{1 x}}{\partial d_{1}} & \frac{\partial F_{1 x}}{\partial \theta_{1}} \ldots \frac{\partial F_{1 x}}{\partial \theta_{1}} \\
\frac{\partial F_{1 y}}{\partial d_{1}} \ldots \frac{\partial F_{1 y}}{\partial d_{1}} & \frac{\partial F_{1 y}}{\partial \theta_{1}} \ldots \frac{\partial F_{1 y}}{\partial \theta_{1}} \\
\frac{\partial F_{1 z}}{\partial d_{1}} \ldots \frac{\partial F_{1 z}}{\partial d_{1}} & \frac{\partial F_{1 z}}{\partial \theta_{1}} \ldots \frac{\partial F_{1 z}}{\partial \theta_{1}} \\
\vdots & \vdots & \vdots \\
\frac{\partial F_{n z}}{\partial d_{7}} & \ldots \frac{\partial F_{n z}}{\partial d_{7}} & \frac{\partial F_{n z}}{\partial \theta_{7}} & \ldots \frac{\partial F_{n z}}{\partial \theta_{7}}
\end{array}\right)_{3 n \times 12} \\
& b=\left(\Delta P_{1 x} \Delta P_{1 y} \Delta P_{1 z} \Delta P_{2 x} \Delta P_{2 y} \Delta P_{2 z} \ldots \Delta P_{n x} \Delta P_{n y} \Delta P_{n z}\right)^{T} \\
& \Delta \mathrm{X}=\left(\begin{array}{llll}
\Delta d_{1} \ldots \Delta d_{7} & \Delta \theta_{1} \ldots \Delta \theta_{7}
\end{array}\right)^{T}
\end{aligned}
$$

The number of measured points should be sufficiently large, which ensures LSM produces an optimal estimation of $\Delta \mathrm{X}$. The estimation is

$$
\Delta \mathrm{X}=\left(\mathrm{A}^{\mathrm{T}} \mathrm{A}\right)^{-1} \mathrm{~A}^{\mathrm{T}} \mathrm{b}
$$

Where $A$ is a function of the seven joint angles, and it's a transformation of regular Jacobian matrix; $b$ is deviation matrix.

\section{KINEMATICS CALIBRATION AND COMPENSATION}

The experiment was carried out using a laser tracker. In the experiment, a target ball was attached to the center of the terminal of the robot. Twenty-five points were measured, in which thirteen points were used for the modeling of error model, and the other twelve points were used for the validation of the calibration. The theoretical D-H parameters of the robot are shown in Table II.

Table II. THEORETICAL D-H PARAMETERS

\begin{tabular}{ccccc}
\hline Link i & $\boldsymbol{\theta}_{\boldsymbol{i}}$ & $\boldsymbol{\alpha}_{\boldsymbol{i}}$ & $\boldsymbol{a}_{\boldsymbol{i}}$ & $\boldsymbol{d}_{\boldsymbol{i}}$ \\
\hline 1 & 0 & -90 & 340 & 0 \\
2 & 0 & 0 & 1200 & 0 \\
3 & 0 & 90 & 192.5 & 0 \\
4 & 0 & -90 & 1020 & 0 \\
5 & 0 & 90 & 138 & 0 \\
6 & 90 & 90 & 0 & 0 \\
7 & -90 & 0 & 0 & 965
\end{tabular}

The approximate calibration provides twist angles and link lengths. The accurate calibration provides the deviation of the other two parameters. The calibrated kinematic parameters, as shown in Table III, are the sum of the theoretical values and the deviations.

Table III. PRACTICAL D-H PARAMETERS

\begin{tabular}{lcccc}
\hline Link i & $\boldsymbol{\theta}_{\boldsymbol{i}}$ & $\boldsymbol{\alpha}_{\boldsymbol{i}}$ & $\boldsymbol{a}_{\boldsymbol{i}}$ & $\boldsymbol{d}_{\boldsymbol{i}}$ \\
\hline 1 & 0.23 & -90.54 & 340.82 & 0.03 \\
2 & 0.20 & 0.02 & 1200.48 & -0.06 \\
3 & 0.18 & 90.05 & 193.87 & 0.04 \\
4 & 0.19 & -90.08 & 1020.14 & 0.08 \\
5 & -0.07 & 89.91 & 138.31 & 0.14 \\
6 & 90.15 & 90.03 & 0.23 & -0.03 \\
7 & -89.94 & 0.02 & 0.15 & 964.12 \\
\hline
\end{tabular}

It's obvious that there are large errors at the terminal of the robot before calibration. This is because of the length and flexibility of all links. A slight deviation on the kinematic parameters will generate a relatively large error at the terminal of the link. As discussed in section III.E, three calibrated components were integrated into the kinematic model recursively to decrease the errors, $\Delta \mathrm{P}$, of position and pose. The components were the compensation for the deformation of structure, the calibrated parameters of links, and the calibrated reduction ratio. Compare the positions of the other twelve points before calibration, Figure , and after calibration, Figure VI, based on the measurement of a laser tracker.

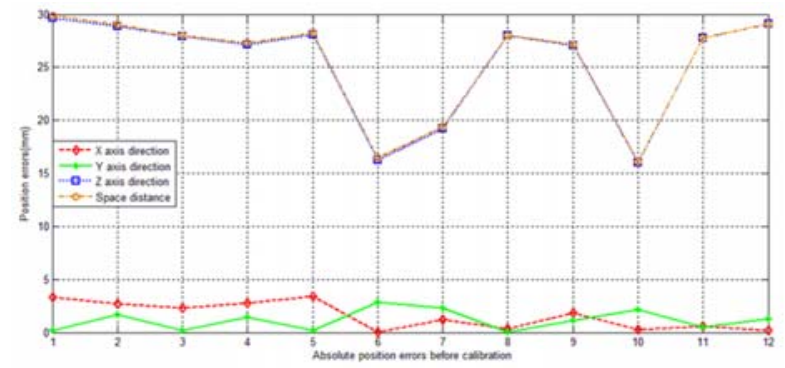

FIGURE VI. ABSOLUTE POSITION ERRORS BEFORE CALIBRATION 


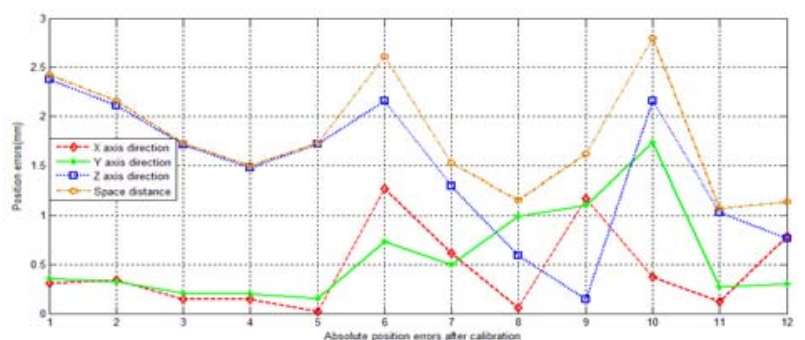

FIGURE VII. ABSOLUTE POSITION ERRORS AFTER CALIBRATION

The comparison shows that the errors along axis $\mathrm{Z}$ are larger than the errors along axes $\mathrm{X}$ and $\mathrm{Y}$. This indicates that the deformation of the links has significant effects on the deviation of the terminal of the robot. The mean error of the absolute position of the twelve points without calibration is $25.42779 \mathrm{~mm}$, and the mean error is reduced to $1.78118 \mathrm{~mm}$ after calibration.

\section{CONCLUSION}

This paper introduced a calibration method based on the multi-parameter kinematic model of a 7-DOF robot. The analysis focused on the deformation of flexible joints and links. The methodology of two-step calibration of kinematic parameters was used to compensate the errors on the kinematic parameters of the robot. The experiment results sufficiently proved that the calibration method, based on multi-parameters and the analysis of the structural deformation, can improve the absolute positioning accuracy of the terminal of the flexible robot. The method is universal to other flexible mechanical equipment for calibration and error compensation.

\section{ACKNOWLEDGMENT}

This research was financially supported by the Science and Technology Program of Tianjin, China (No.16YFZCGX00040).

\section{REFERENCES}

[1] Li Ding kun, Ye Sh eng hua, Re n Yo ng jie, et a l. Research on robot's positioning accuracy calibration[J]. Ac ta Metrologica Sinica, 2007, 28( 3): 224-227.

[2] Mooring B W, Roth Z S, Driels M R. Fundamentals of manipulator calibration[M]. New York, USA: Wiley, 1991.

[3] Roth Z S, Mooring B W, Ravani B. An overview of robot calibration[J]. IEEE Journal of Robotics and Automation, 1987,3(5): 377-385.

[4] LIU Zhi, ZHAO Zhengda, XIE Ying, et al, Kinematic Calibration and Compensation for a Robot with Structural Deformation[J]. Robot, 2015, 37(3): 376-384.

[5] Kazerounian K, Qian G Z. Kinematic calibration of robotic manipulators[J]. ASME Journal of Mechanisms, Transmission andAutomation in Design, 1989, 111(4): 482-487.

[6] Dumas C, Caro S, Cherif M, et al. Joint stiffness identification of industrial serial robots[J]. Robotica, 2012, 30(4): 649-659.

[7] Meggiolaro M A, Dubowsky S, Mavroidis C. Geometric and elastic error calibration of a high accuracy patient positioning system[J]. Mechanism and Machine Theory, 2005, 40(4): 415-427.

[8] Lightcap C, Hamner S, Schmitz T, et al. Improved positioning accuracy of the PA10-6CE robot with geometric and flexibility calibration[J]. IEEE Transactions on Robotics, 2008, 24(2): 452-456.

[9] Wang Y, Liu C J, Ren Y J, et al. Compensation for positioning error of industrial coordinate measurement robot[J]. Journal of Mechanical Engineering, 2011, 47(15):
[10] Fernández-Bustos I, Agirrebeitia J, Ajuria G, et al. A new finite element to represent prismatic joint constraints in mechanisms[J]. Finite Elements in Analysis \& Design, 2007, 43(1): 36-50.

[11] Deblaise D, Hernot X, Maurine P. A systematic analytical method for PKM stiffness matrix calculation[C]// IEEE International Conference on Robotics and Automation. IEEE, 2006: 4213-4219. 